\title{
All the studies on hyperprolactinemia should not forget to consider the possible presence of macroprolactinemia
}

\author{
Pierluigi Amadori, Costantino Dilberis ${ }^{1}$ and Alberto Marcolla ${ }^{2}$ \\ Outpatient Endocrinology Service, Azienda Provinciale per i Servizi Sanitari, Trento, Italy, ${ }^{1}$ Laboratory of Clinical Analysis, S. Camillo Hospital, Trento, \\ Italy and ${ }^{2}$ Clinical Chemistry Unit, Azienda Provinciale per i Servizi Sanitari Hospital, Cles, Trento, Italy
}

(Correspondence should be addressed to P Amadori, Ambulatorio di Endocrinologia, Azienda Provinciale per i Servizi Sanitari, Piazza Venezia 41, 38100 Trento, Italy; Email: amadoripierluigi@libero.it)

Prolactin (PRL) in human serum has been classified into three main species on the basis of molecular mass: monomeric PRL, big PRL and big, big PRL (bb-PRL), called 'macroprolactin', with molecular masses of $23 \mathrm{kDa}, 50-60 \mathrm{kDa}$ and $150-170 \mathrm{kDa}$ respectively (1). Although the nature of bb-PRL is heterogeneous, the most common form of macroprolactin is a complex of PRL and immunoglobulin G $(2,3)$. Most patients with macroprolactinemia do not show any clinical symptoms of hyperprolactinemia, such as amenorrhea, galactorrhea and infertility, despite high hyperprolactinemic levels and they do not need specific treatment $(4-6)$.

Macroprolactin is recognized, in various degrees, by immunoassays for PRL $(6,7)$ and has a slower clearance from serum than PRL, causing diagnostic confusion in evaluating hyperprolactinemic conditions. The incidence of macroprolactinemia ranges from $15 \%$ up to $26 \%$ of all hyperprolactinemic sera $(4,5)$ and represents the main cause of interassay variability for PRL dosage (7).

Methods for big and bb-PRL detection are gel filtration chromatography, sensitive and specific but expensive (1), and polyethylene glycol (PEG) precipitation, a screening test for bb-PRL, not applicable to all immunometric assays $(5,6,8)$.

In their interesting work 'Gender differences in the prevalence, clinical features and response to cabergoline in hyperprolactinemia' (9), Colao et al. seem not to have evaluated the possibility of macroprolactinemia being a cause of hyperprolactinemia among their patients. Considering the very high PRL levels and the numerous clinical manifestations, macroprolactinemia is very unlikely in the 'macroprolactinoma' groups, but it cannot be excluded in either the 'microprolactinoma' or the 'non-tumoral' groups. Interestingly, $24 \%$ and $20 \%$ of the women included in the latter two groups, respectively, did not show menstrual disturbances (i.e. they were eumenorrhoeic) and this is very suggestive of macroprolactinemia.

In our recent study (10), we noted that macroprolactinemia is actually the main cause of persistent hyperprolactinemia in eumenorrhoeic women, being present in 14 out of 15 cases. All these patients had normal (monomeric) PRL concentrations after macroprolactin precipitation with PEG. Although macroprolactinemia has been considered to be a cause of apparent resistance to antiprolactinemic drugs $(4,11)$, neither PRL normalization on dopaminergic treatment nor the presence of a pituitary mass can exclude macroprolactinemia, the former varying from $100 \%$ (12) to $46 \%$ (13). As for the latter, macroprolactinemia has been quite often associated with pituitary adenomas, probably not prolactinomas $(3,10,11)$.

Colao and coworkers (9) report a decrease in PRL levels from $144 \pm 62$ to $14.4 \pm 21.5 \mu \mathrm{g} / \mathrm{l}$ in microprolactinomas with cabergoline treatment (9). However, the graphics in their Fig. 2 show that some patients with basal PRL close to $100 \mu \mathrm{g} / \mathrm{l}$ have similar values after 6 months of therapy. It would be interesting to check whether these patients overlap the eumenorrhoeic women classified in the 'microprolactinoma' group. If so, the presence of macroprolactinemia would be highly probable.

Finally, we consider that every study concerning hyperprolactinemia should look carefully for the presence of macroprolactin, its amount and hence the monomeric PRL concentrations. Otherwise, patients who are not 'pathologically' hyperprolactinemic may be classified as such, causing an important bias.

\section{References}

1 Smith CR \& Norman MR. Prolactin and growth hormone: molecular heterogeneity and measurement in serum. Annals of Clinical Biochemistry 199027 542-550.

2 Lindstedt G. Endogenous antibodies against prolactin - a 'new' cause of hyperprolactinemia. European Journal of Endocrinology $1994130429-432$.

3 De Schepper J, Schiettecatte J, Velkeniers B, Blumenfeld Z, Shteinberg M, Devroey P et al. Clinical and biological characterization of macroprolactinemia with and without prolactin-IgG complexes. European Journal of Endocrinology $2003 \mathbf{1 4 9}$ 201-207.

4 Hattori N, Ikekubo K, Ishihara T, Moridera K, Hino M \& Kurahachi H. Correlation of the antibody titers with serum prolactin levels and their clinical course in patients with 
anti-prolactin autoantibody. European Journal of Endocrinology $1994130438-445$.

5 Leslie H, Courtney CH, Bell PM, Hadden DR, McCance DR, Ellis PK et al. Laboratory and clinical experience in 55 patients with macroprolactinemia identified by a simple polyethylene glycol precipitation method. Journal of Clinical Endocrinology and Metabolism 200186 2743-2746.

6 Cavaco B, Prazeres S, Santos MA, Sobrinho LG \& Leite V. Hyperprolactinemia due to big big prolactin is differently detected by commercially available immunoassays. Journal of Endocrinological Investigation 199922 203-208.

7 Smith TP, Suliman AM, Fahie-Wilson MN \& McKenna TJ. Gross variability in the detection of prolactin in sera containing big big prolactin (macroprolactin) by commercial immunoassays. Journal of Clinical Endocrinology and Metabolism $2002 \mathbf{8 7}$ 5410-5415.

8 Fahie-Wilson M, Brunsden P, Surrey J \& Everitt A. Macroprolactin and the Roche Elecsys prolactin assay: characteristics of the reaction and detection by precipitation with polyethylene glycol. Clinical Chemistry 200046 1993-1995.

9 Colao A, Di Sarno A, Cappabianca P, Briganti F, Pivonello R, Di Somma $\mathrm{C}$ et al. Gender differences in the prevalence, clinical features and response to cabergoline in hyperprolactinemia. European Journal of Endocrinology 2003148 325-331.
10 Amadori PL, Dilberis C, Marcolla A, Pinamonti M, Menapace P \& Dal Bosco F. Macroprolactinemia: predictability on clinical basis and detection by PEG precipitation with two different immunometric methods. Journal of Endocrinological Investigation 200326 148-156.

11 Cavaco B, Leite V, Santos MA, Arranhado E \& Sobrinho LG. Some forms of big big prolactin behave as a complex of monomeric prolactin with an immunoglobulin $\mathrm{G}$ in patients with macroprolactinemia or prolactinoma. Journal of Clinical Endocrinology and Metabolism $1995802342-2346$.

12 Olukoga AO \& Kane JW. Macroprolactinaemia: validation and application of the polyethylene glycol precipitation test and clinical characterization of the condition. Clinical Endocrinology $199951119-126$.

13 Vallette-Kasic S, Morange-Ramos I, Selim A, Gunz G, Morange S, Enjalabert M et al. Macroprolactinemia revisited: a study on 106 patients. Journal of Clinical Endocrinology and Metabolism 200287 $581-588$.

Received 19 September 2003

Accepted 22 September 2003 